\title{
TTR
}

Traduction, terminologie, re?daction

\section{Les noms propres et leurs dérivés dans le vocabulaire de l'intelligence artificielle}

\section{Monique C. Cormier et Jean Fontaine}

Volume 8, numéro 2, 2e semestre 1995

Technolectes et dictionnaires

URI : https://id.erudit.org/iderudit/037220ar

DOI : https://doi.org/10.7202/037220ar

Aller au sommaire du numéro

Éditeur(s)

Association canadienne de traductologie

ISSN

0835-8443 (imprimé)

1708-2188 (numérique)

Découvrir la revue

Citer cet article

Cormier, M. C. \& Fontaine, J. (1995). Les noms propres et leurs dérivés dans le vocabulaire de l'intelligence artificielle. TTR, 8(2), 103-149.

https://doi.org/10.7202/037220ar
Résumé de l'article

Les noms propres et leurs dérivés dans le vocabulaire de l'intelligence artificielle - Cet article présente les résultats d'une étude qui porte sur un sujet relativement peu exploré par les terminologues, à savoir la part des noms propres et de leurs dérivés dans la formation des vocabulaires techniques et scientifiques. Nous nous intéressons en particulier dans cet article aux onomastismes qui font partie du vocabulaire de l'intelligence artificielle. L'article se divise en trois parties. Nous présentons tout d'abord les matrices morphosyntaxiques qui composent les onomastismes de l'intelligence artificielle. Nous étudions ensuite les unités terminologiques complexes onomastiques du point de vue du nom propre et de son réfèrent. Enfin, nous examinons ces mêmes unités du point de vue de leur déterminé.
Tous droits réservés (C TTR: traduction, terminologie, rédaction — Les auteurs, 1995
Cedocument est protégé par la loi sur le droit d'auteur. L'utilisation des services d'Érudit (y compris la reproduction) est assujettie à sa politique d'utilisation que vous pouvez consulter en ligne.

https://apropos.erudit.org/fr/usagers/politique-dutilisation/ 


\section{Les noms propres et leurs dérivés dans le vocabulaire de l'intelligence artificielle}

\section{Monique C. Cormier Jean Fontaine}

\section{Introduction}

Les mots de la langue courante dérivés de noms propres ont fait l'objet de nombreuses études. Précisons d'emblée, à des fins de clarté, que ce phénomène a reçu, selon des auteurs, différentes appellations, notamment celles d' "onomastisme», de "formation déonomastique», ou encore d'(éponyme», avec des définitions qui ne se recoupent pas toujours exactement. Ainsi, pour Jean-Claude Boulanger (1986, pp. 8687), l'onomastisme est un terme générique qui désigne tout dérivé ou composé de nom propre (lieu, personne, etc.). Il comprend deux spécifiques: anthroponymisme, qui désigne tout dérivé ou composé de nom de personne (anthroponyme), et toponymisme, qui désigne tout dérivé ou composé de nom de lieu (toponyme). Lorsque le toponymisme sert à dénommer les habitants d'un lieu, il s'agit d'un gentilé. À ces deux principaux sous-groupes, on pourrait en outre ajouter les dérivés ou composés de noms d'institutions, de groupes politiques, etc. (sorbonnard, normalien, onusien, péquiste) et les dérivés ou composés de marques déposées (xérographie, scrabbleur, walkmanie). Pour sa part, Wolfgang Schweickard (1989, p. 242) definit la «formation déonomastique» comme un mot dérivé d'un nom propre, qu'il y ait changement morphologique (Sade $\rightarrow$ sadisme) ou non (Braille $\rightarrow$ braille). Enfin, André Lapierre $(1989$, p. 588$)$ utilise le terme "éponyme» pour désigner tout dérivé de nom propre (de personne, de lieu) ou de nom propre «communisé». Il observe également que l'équivalent anglais 
«eponym» est bien établi dans la lexicographie anglaise moderne, bien qu'on semble exclure de la définition d'ueponym» les dérivés de noms de lieux. Comme on peut le constater, il existe un flou terminologique dans la désignation du phénomène linguistique que représentent ces mutations linguistiques.

Comme nous le précisions plus tôt, les mots de la langue générale dérivés de noms propres ont fait l'objet de nombreuses analyses et ces dernières ont, pour ce qui est du français, surtout porté sur les formations simples (unités isolées). Du côté des langues de spécialité, rares sont les auteurs qui se sont penchés sur le statut du nom propre ou de ses dérivés. Nous pouvons citer Henri Van Hoof (1986), qui s'est intéressé aux éponymes dans le domaine médical, et qui est d'ailleurs l'un des seuls, à notre connaissance, à avoir traité des unités lexicales complexes onomastiques dans une langue de spécialité. Si les recherches sur les unités lexicales complexes en terminologie sont abondantes', les recherches sur les unités lexicales complexes onomastiques, toujours en terminologie, sont elles, à peu près inexistantes.

Pour leur part, les onomasticiens se sont intéressés à ces constructions, particulièrement dans l'optique toponymique (ex.: générique + spécifique $\rightarrow$ nom de l'entité géographique + nom propre: baie de Gaspé). Le résultat de cette association est un syntagme dont la fonction est individualisante, dénominative. Il n'y a qu'une baie de Gaspé, c'est une entité unique qui s'apparente à un autre nom propre. Ces constructions s'opposent à celles qui font l'objet de la présente recherche. Celles-ci demeurent des représentantes de classe, c'est-à-dire des notions. Ainsi, loi de Hubble est un terme désignant un concept tandis que comète de Halley identifie un objet unique parmi toutes les comètes de l'univers. Les travaux des onomasticiens diffèrent des nôtres en ce sens qu'ils s'intéressent davantage à l'aspect identitaire du nom propre ou de ses dérivés alors que nos préoccupations sont plutôt de type classificatoire. De la même façon, ils s'intéressent beaucoup à l'aspect diachronique alors que c'est plutôt, dans le cadre de la présente recherche, l'aspect synchronique qui retient davantage notre attention.

1. Voir Boulanger et Nakos (1988). 
Les résultats que nous présentons portent sur un sujet relativement peu exploré par les terminologues, soit la part des noms propres et de leurs dérivés dans la formation des vocabulaires techniques et scientifiques. Nous nous intéresserons en particulier aux onomastismes qui font partie du vocabulaire de l'intelligence artificielle. Notre article se divisera en trois parties. Nous présenterons tout d'abord les matrices morphosyntaxiques complexes qui composent les onomastismes de l'intelligence artificielle. Puis, nous étudierons les unités terminologiques complexes onomastiques du point de vue du nom propre et de son référent. Enfin, nous examinerons ces mêmes unités du point de vue de leur base.

Le corpus qui a fait l'objet de l'étude réunit les termes provenant de trois sources distinctes, soit la nomenclature française du Vocabulaire de l'intelligence artificielle, de Noël Lazure, publié en 1988 par le Secrétariat d'État du Canada, celle du Vocabulaire d'intelligence artificielle, de Gabriel Otman, publié en 1991 par le Centre de terminologie et de néologie de l'INaLF-CNRS, et celle que mon collègue Jacques Lethuillier et moi-même avons publiée sous forme de lexique anglais-français dans la revue Meta en 1990 et 1991 . Réunies en un seul grand corpus et une fois les doublets et triplets éliminés, ces trois nomenclatures comptent un total de 14394 termes, dont $1,2 \%$, soit 176 termes, est constitué de noms propres ou de dérivés de noms propres.

\section{Les matrices morphosyntaxiques complexes ${ }^{2}$}

De ces 176 termes, seuls cinq sont des termes simples; tous les autres sont des unités terminologiques complexes et seules trois matrices les composent, c'est-à-dire la forme $\mathrm{N}$ prép. $\mathrm{X}$, la forme $\mathrm{N}$ joncteur $ø \mathrm{X}$ et la forme $\mathrm{N} X$-ien(ne), dans lesquelles $\mathrm{N}=$ nom, $X=$ nom propre, prép = prépositions (de, du, des), et $X$-ien(ne), le dérivé de nom propre. Le Tableau 1 (voir Annexe) présente les diverses matrices terminologiques

2. La thématique de la présente section a fait l'objet d'une communication lors du XVIII ${ }^{e}$ Congrès international des sciences onomastiques qui s'est tenu à Trèves, en Allemagne, en avril 1993 et le texte sera publié dans les Actes. 
relevées avec, pour chacune, le nombre de termes correspondants et un exemple.

Ce tableau a été divisé en quatre groupes principaux:

1. Les termes simples ( 5 sur $176=3 \%$ );

2. La forme N prép. X (ex.: suite de Haming) avec ses variantes (92 sur $176=52 \%$ );

3. La forme $\mathrm{N}$ joncteur $\varnothing \mathrm{X}$ (ex.: analyseur Marcus) et ses variantes (11 sur $176=6 \%$ );

4. La forme $\mathrm{N} \mathrm{X}$-ien(ne) (ex.: chaîne eulérienne) et ses variantes (68 sur $176=39 \%$ ).

Les deux formes les plus fréquentes sont donc $\mathrm{N}$ de $\mathrm{X}$ ( 74 termes sur 92) et $\mathrm{N} X$-ien(ne) (60 termes sur 68) et elles constituent à elles seules plus des trois quarts des 176 onomastismes.

Dans quelques cas seulement, deux formes concurrentes se rencontrent pour le même terme (cercle d'Euler / cercle eulérien). Nous reviendrons sur cette question.

\subsection{La forme $\mathrm{N}$ prép. $\mathrm{X}$}

Plus de la moitié ( $52 \%$ ) des onomastismes relevés est donc du type $\mathrm{N}$ prép. X (suite de Haming) ou en est une variante. Dans tous les cas, la préposition est de, sauf pour algorithme du British Museum, où la nature de ce nom propre - il s'agit d'une institution - rend obligatoire l'utilisation de l'article partitif. Les variantes (voir le Tableau 1), somme toute assez peu nombreuses, sont des unités complexes où un adjectif est postposé (réseau de Pétri temporisé), des syntagmes nominaux complexes (algorithme de transformée de Fourier; système de production de Post), ou encore des unités complexes à deux anthroponymes associés (procédure de Knuth et Bendix).

\subsection{La forme $\mathrm{N}$ joncteur $ø \mathrm{X}$}

Quant à la forme $\mathrm{N}$ joncteur $ø \mathrm{X}$, elle se retrouve dans $6 \%$ des onomastismes relevés, soit 11 cas où l'unité terminologique correspond à la combinaison de deux noms dont le second est un nom propre 
(analyseur Marcus) et donc où tout morphème de liaison est ou a été effacé. Dans plusieurs de ces cas, qui s'apparentent à l'apposition, le nom propre représente un nom de produit, de marque déposée (langage informatique, programme, logiciel, etc.): compilateur Ada, logique Pascal. Mais ce n'est pas le cas pour d'autres (voisinage Golay, méthode Monte Carlo). Ces résultats semblent différer des observations que fait Bernard Quemada (1978, p. 1185) au sujet de la construction des dénominations techniques, lorsqu'il dit:

L'emploi d'un nom propre (ou de l'adjectif correspondant) en matière d'élément spécificateur est depuis la fin du XVII siècle un usage caractéristique des langues techniques. La construction prépositionnelle, plus classique et mieux conservée dans les dénominations scientifiques moins banalisées (thermomètre et balance de Bernoulli, de Réaumur ou de Roberval), est moins fréquente aujourd'hui que la construction directe à partir de noms de personnes, inventeurs ou parrains: lampe Davy,procédé Solvay, vernis Martin [...].

\subsection{La forme N X-ien(ne)}

Parmi les unités terminologiques relevées, 68 , soit $39 \%$ des onomastismes, renferment des adjectifs dérivés de noms de personnes, la dérivation se faisant dans presque tous les cas au moyen du formant -ien(ne). Plusieurs auteurs ${ }^{3}$ ont déjà noté, pour la langue générale, que le formant -ien est le plus fréquent dans la dérivation à partir de noms propres, ce qui semble être également le cas en langue de spécialité, si on se fie à nos résultats. Voici donc ces adjectifs et, pour chacun, le nombre d'unités terminologiques complexes qu'ils composent:

$\begin{array}{lc}\text { aristotélicien } & 2 \\ \text { bayésien } & 11 \\ \text { booléen } & 24 \\ \text { diophantien } & 1 \\ \text { euclidien } & 3\end{array}$

3. Notamment Anker Teilgård Laugesen (1974, p. 261), René de Chantal (1961, p. 178) et Jean-Yves Dugas (1982, p. 28 et 1986, p. 239). 


$\begin{array}{ll}\text { eulérien } & 8 \\ \text { gaussien } & 5 \\ \text { hamiltonien } & 7 \\ \text { Knuthien } & 1 \\ \text { lagrangien } & 1 \\ \text { markovien } & 1 \\ \text { shefferien } & 1 \\ \text { socratique } & 3\end{array}$

Même dans le vocabulaire courant, ce formant est privilégié lorsqu'il s'agit de dériver d'un nom de personne un adjectif de connotation neutre, indiquant une simple relation avec la personne (kantien, balzacien, beethovénien). Des dérivés de noms d'auteurs ${ }^{4}$ peuvent prendre, dans certains contextes, une connotation particulière: ainsi, kafkaïen, outre sa référence directe «qui a rapport à Kafka», peut prendre le sens de «qui rappelle l'atmosphère oppressante des romans de Kafka». Le formant -ien est aussi très utilisé pour forger des gentilés à partir de toponymes (canadien, norvégien, parisien).

Si l'on revient à notre étude, on constate que non seulement -ien et sa variante -éen sont privilégiés pour former des onomastismes, mais qu'ils sont presque réservés à cet usage dans l'ensemble du corpus de 14394 termes. En effet, il existe seulement 2 occurrences où ce formant ne sert pas à créer un onomastisme: heuristique échiquéenne (du jeu d'échecs) et syntaxe lispienne (de LISP, acronyme pour list processing language). Dans ce dernier cas, on pourrait même à la rigueur considérer LISP comme un nom propre, une sorte de marque déposée, et donc lispien comme un dérivé de nom propre.

Examinons maintenant le double mode de formation des adjectifs du corpus au moyen du suffixe -ien, ou de sa variante -éen, et du suffixe -ique.

4. Pour d'autres considérations sur les dérivés de noms d'auteurs, voir notamment René de Chantal (1961), Colette Monsarrat (1974), Anker Teilgård Laugesen (1974), Marek Gawełko (1977 et 1977a) et Jean-Yves Dugas (1982). 
Dans le premier mode, on retrouve la base (nom propre) intacte + le suffixe. Ainsi, le formant -ien s'ajoute souvent au nom propre terminé par une consonne sans autre modification de celui-ci, si ce n'est la perte de la majuscule à l'initiale:

$\begin{array}{lll}\text { Gauss } & \rightarrow & \text { gaussien } \\ \text { Hamilton } & \rightarrow & \text { hamiltonien } \\ \text { Markov } & \rightarrow & \text { markovien } \\ \text { Sheffer } & \rightarrow & \text { shefferien }\end{array}$

Dans le deuxième mode de formation des adjectifs, tout en constatant la perte de la majuscule à l'initiale, on retrouve la base (nom propre), de laquelle est retranché la voyelle finale, ici le $-e,+$ le suffixe. Ainsi, il y a substitution, le suffixe -ien prenant la place du $-e$ :

$\begin{array}{lll}\text { Diophante } & \rightarrow & \begin{array}{l}\text { diophantien } \\ \text { Euclide }\end{array} \\ \text { Lagrange } & \rightarrow & \text { lagrangien }\end{array}$

Enfin, quelques cas particuliers retiennent l'attention. D'abord, l'adjectif dérivé Knuthien, le seul du corpus français à conserver la majuscule initiale, provient du terme sémantique Knuthienne (Lazure 1988, pp. 347 et 1108). La présence de la majuscule est peut-être une simple coquille dans le texte source ou une fantaisie. Anker Teilgård Laugesen (1974, p. 258), dans un tout autre contexte, a relevé l'utilisation sporadique de la majuscule chez un même critique littéraire (la conception Pascalienne, les réflexions Pascaliennes à côté de la mystique pascalienne, les fragments pascaliens), mais en général une telle pratique est rare, car elle contrevient à l'orthographe. Il se peut également que l'on retrouve ici l'influence de l'anglais, l'équivalent en étant Knuthian semantics, et l'usage anglais ne semblant pas fixé en ce qui a trait à l'utilisation de la majuscule pour de tels adjectifs, en tout cas dans le domaine de l'intelligence artificielle. Autre cas, celui de voyellesgraphiquementaccentuées:Bayes $\rightarrow$ bayésien, Euler $\rightarrow$ eulérien. La syllabe fermée du nom propre s'ouvre par l'adjonction du suffixe, ce qui entraîne l'accent graphique sur le $-e$. Mais ce procédé n'est pas systématique. À côté de Euler $\rightarrow$ eulérien, nous avons vu plus haut Sheffer $\rightarrow$ shefferien, sans accent graphique. Anker Teilgård Laugesen (1974, p. 270) fait la même observation: "Voyelle finale accentuée 
suivie d'une consonne prononcée: le passage phonétique de [è] à [é], dû au déplacement de l'accent, entraîne le changement graphique de è, e en é: (...) Jaurès $\rightarrow$ jaurésien (...) Adler $\rightarrow$ adlérien. Pourtant, on écrit bien schopenhauerien et heideggerien.»

La variante suffixale -éen, quant à elle, n'apparaît que pour un adjectif du corpus:

Boole $\rightarrow \quad$ booléen

La voyelle finale du nom anglais Boole étant muette, on se serait normalement attendu à la forme boolien. Et, effectivement, les formes plus rares boolien et même booléien sont aussi attestées, dans certains dictionnaires français. Anker Teilgård Laugesen (1974, p. 268) dit d'ailleurs à ce propos:

Tandis que les noms anglais subissent le même sort que les noms français [la finale muette $-e$ tombe sur le plan graphique], les noms allemands gardent leur finale inaccentuée mais tout de même audible, -e sous forme de -é, donc distinctement prononcée: en un mot, on retombe alors dans le cas de mallarméen: Goethe $\rightarrow$ goethéen, Nietzsche $\rightarrow$ nietszchéen.

La forme booléen est sans doute un calque de l'anglais boolean. Cet exemple soulève à nouveau la question de savoir, lorsque le nom propre à partir duquel il y a dérivation est étranger, si l'adjectif dérivé est entré dans la langue française par dérivation ou bien par le biais d'un calque sur une forme étrangère. Ainsi, l'adjectif hamiltonien est-il un véritable dérivé français de Hamilton ou bien est-il un simple calque de l'adjectif anglais hamiltonian? Dans ces cas, seules les datations peuvent aider à trouver l'étymon véritable.

Quant à l'adjectif dérivé aristotélicien, inclus dans les autres dérivés en -ien, il ne provient pas directement du nom Aristote, mais d'un autre adjectif, aristotélique, qu'il a supplanté au XVII' siècle, selon l'évolution suivante (Rey 1992, p. 111):

gr. Aristotelês $\rightarrow$ lt. aristotelicus $\rightarrow$ aristotélique (1527): par emprunt savant 
par dérivation savante.

Le formant -ique (gr. -ikos, lat. -icus), quant à lui, est moins utilisé en français pour produire des dérivés d'anthroponymes. Le seul adjectif de ce type dans le corpus est socratique, dont l'origine est la suivante (Rey 1992, p. 1959):

gr. sôkratikos (emprunt au grec) $\rightarrow$ lat. socraticus (emprunt au latin) $\rightarrow$ fr. socratique (1540).

Enfin, un seul substantif du corpus a été obtenu par suffixation, au moyen de -isation, dérivé du formant verbal savant -iser (lat. ittiare), qui date de la Renaissance. Il s'agit du terme skolémisation.

Skolem $\rightarrow \quad$ skolémisation

Notons que la forme verbale "skolémiser est absente du corpus.

\subsection{Unités terminologiques plus complexes en X-ien}

Quelques unités terminologiques renfermant des adjectifs en -ien sont des variantes plus complexes de la forme de base $\mathrm{N} \mathrm{X-ien(ne).} \mathrm{Nous}$ avons le cas intéressant de l'unité graphe non hamiltonien, où l'opposition hamiltonien / non hamiltonien suppose un sens bien précis à l'adjectif, qui dépasse la simple relation «dû à Hamilton». Un exemple plus connu de ce type est celui des géométries non euclidiennes. Ainsi, une géométrie (non) euclidienne n'est pas une géométrie due (ou non) à Euclide, mais une géométrie fondée (ou non) sur le postulat des parallèles formulé par Euclide. Le sens de géométrie euclidienne n'est pas compositionnel et géométrie euclidienne n'égale donc pas nécessairement géométrie d'Euclide.

Dans le cas de dispositif de reconnaissance bayésien, on constate que l'adjectif dérivé de nom propre peut déterminer un syntagme nominal aussi bien qu'un simple substantif. Même chose avec bruit additif gaussien, où l'on remarque que l'adjectif dérivé d'un nom propre suit un autre adjectif. Marek Gawełko (1977, p. 124) précise, à 
propos de l'adjectif dérivé de noms d'auteurs, et faisant référence à des exemples donnés par Anker Teilgård Laugesen (1974, p. 259):

L'emploi de l'adjectif est en général aussi exclu lorsque le substantif est déjà suivi d'un autre adjectif: les pensées pascaliennes, mais les pensées tragiques de Pascal. Nous ne savons pas pourquoi le français évite la rencontre de deux adjectifs postposés au substantif; ce qui est sûr c'est que l'adjectif concrétise le substantif, parfois au point que celui-ci ne peut plus désigner qu'un exemplaire donné: le texte même de Pascal.

Notre exemple nuance ces constats. Mais la différence entre bruit additif gaussien et pensées tragiques de Pascal réside dans la nature de l'adjectif intercalé (relationnel dans le premier cas; qualificatif dans le second) et le fait que la seconde unité est de nature accidentelle, discursive et non pas figée, c'est-à-dire terminologique, comme dans le premier exemple.

Le corpus comprenait également une unité pour le moins inusitée: littéral de booléen (Lazure, 1988, pp. 65 et 937). L'équivalent anglais donné est boolean litteral. On s'attendrait à voir plutôt littéral booléen en français. Précisons qu'ici littéral est bien un substantif.

\section{Cas particuliers d'onomastismes}

\subsection{Onomastismes composés de plusieurs noms propres}

Le corpus étudié comprend 6 cas où deux anthroponymes $\mathrm{X}$ et $\mathrm{Y}$ sont réunis dans le même terme: forme de Backus Naur, notation de Backus Naur, algorithme de Knuth et Bendix, procédure de Knuth et Bendix, hypothèse de Sapir-Whorf, hypothèse Sapir-Whorf.

Ce nombre est relativement peu élevé lorsqu'on le compare, par exemple, à celui des onomastismes dans le domaine médical, où abondent les termes composés de deux ou trois anthroponymes: noud de Keith et Flack, bacille de Calmette-Guérin, épilepsie BravaisJacksonienne, syndrome de Charcot-Weits-Barber (exemples tirés de Henri Van Hoof 1986, pp. 62, 63, 68). On peut constater dans ces formes lourdes le souci de reconnaître la contribution (indépendante ou en collaboration) de plusieurs chercheurs à telle découverte. À une 
époque où la science n'est plus l'affaire de savants isolés, ni de frontières, ce type d'unités devrait continuer de proliférer. On pourrait suivre l'exemple de cet important collectif de mathématiciens français qui s'est donné un pseudonyme, Nicolas Bourbaki. On en a tiré l'adjectif bourbakiste (parfois écrit bourbachiste).

Les querelles de priorité, d'attribution de la découverte et le chauvinisme contribuent souvent, quand ce n'est pas à la juxtaposition de plusieurs anthroponymes dans le même terme, à la création d'onomastismes concurrents. L'historien des sciences Michel Authier $(1989$, p. 265$)$ constate, à propos de la loi de la réfraction que les Français attribuent à Descartes, ce qui suit:

Il faut être un Français du XX $X^{\mathfrak{e}}$ siècle pour ne pas savoir qu'aux alentours de 1620 le Hollandais Willebrord Snell, dit Snellius (1591-1626), couronnait l'édifice keplérien en établissant la loi de la réfraction qui porte aujourd'hui son nom dans tous les autres pays du monde. Aux dernières nouvelles la guerre fait toujours rage. Dans l'article Descartes (loi de) du Grand Larousse encyclopédique le nom de Snell est absent, tout comme celui de René Descartes dans l'article "Snell's law" de l'Encyclopaedia Britannica.

\subsection{Toponymes}

Dans le lexique courant, même si on exclut les simples gentilés et adjectifs relationnels, les noms de personnes semblent fournir beaucoup plus d'onomastismes que les noms de lieux (Lapierre 1989, p. 592). Parmi les 176 onomastismes, seulement 6 proviennent d'un toponyme plutôt que d'un anthroponyme: algorithme du British Museum, procédure British Museum, syntaxe Edinburgh, méthode Monte Carlo, tour de Hanoi, conférence de Dartmouth.

L'origine des deux premiers termes, qui sont synonymes, diffère selon les sources consultées:

British Museum. Procédure de résolution dénuée d'intelligence, qui explore systématiquement tous les états obtenus, leur applique tous les opérateurs, etc. jusqu'à l'apparition de la solution. (...) Nota: Ainsi appelée par analogie à l'efficacité d'une méthode qui 
consisterait à attendre que les singes pressant au hasard les touches d'une machine à écrire, reproduisent les titres de livres de la bibliothèque du British Museum. (Pavel 1989, p. 44)

Plus vraisemblable paraît l'explication qui suit:

La procédure British Museum inspecte tous les chemins. Une technique pour trouver le chemin le plus court dans un réseau consiste à rechercher tous les chemins possibles, puis à ne conserver que le plus court. Cette procédure est connue sous le nom ironique de procédure du British Museum ${ }^{5}$. (...)

${ }^{5}$ Le British Museum est l'un des plus célèbres musées londoniens et est réputé pour l'incroyable complexité de ramification de ses galeries. Il n'est pas rare qu'un visiteur désirant visiter telle section du musée soit obligé de parcourir toutes les galeries avant de trouver celle qui l'intéresse. D'où le nom donné à la procédure, que l'on appelle aussi en français procédure de l'énumération explicite. (N.d.T.) (Winston 1988, p. 99)

Dans le cas de syntaxe Edinburgh, il a été impossible de retracer l'origine et nous l'avons classé parmi les toponymes, puisque nous n'avons trouvé aucun nom de chercheur Edinburgh.

La méthode Monte Carlo évoque ce quartier de Monaco et son célèbre casino. Le terme désigne en effet une méthode faisant appel au hasard: «A method of obtaining an approximate solution to a numerical problem by the use of random numbers.» (Lazure, 1988, p. 411)

La tour de Hanoi est un jeu d'origine chinoise, formé de trois bâtons et d'une pile d'anneaux de diamètre décroissant qu'il s'agit de transférer d'un bâton à un autre, en respectant certaines règles. Nous n'avons pu trouver si le jeu provient effectivement d'Hanoi ou si c'est sa forme qui rappelle un monument de cette ville au Viêtnam. Notons qu'un autre jeu populaire auprès des chercheurs en intelligence artificielle est aussi un toponyme: le problème des ponts de Königsberg. Il s'agit d'un problème qui fut résolu au XVIII ${ }^{e}$ siècle par le mathématicien Euler qui prouva qu'il était impossible de parcourir en un circuit fermé les sept ponts de cette ville (rebaptisée Kaliningrad, en Russie), sans passer deux fois par le même pont. Le problème est aussi 
connu sous le nom de problème d'Euler et, en théorie des graphes, un tel chemin s'appelle un cycle eulérien.

Enfin, le terme conférence de Dartmouth, que l'on peut considérer comme peu lexicalisé, correspond à la conférence de 1956, c'est-à-dire le congrès où naquit l'intelligence artificielle (et où d'ailleurs le terme anglais artificial intelligence aurait été utilisé pour la première fois).

À la limite, on aurait pu classer le terme algorithme dans les toponymes, puisque ce mot provient du surnom d'un mathématicien arabe, $A l$ Huwarizmi, surnom qui est en même temps un gentilé, puisqu'il dérive lui-même du nom de la région d'Asie centrale appelée Huwarizm.

\section{Les onomastismes du point de vue de X: le nom propre et son référent}

On peut se demander si la forme que prend un onomastisme peut dépendre de la personne portant le nom propre d'origine ou du nom luimême. L'intérêt historique de la chose s'y ajoutant, nous avons recueilli quelques données biographiques sommaires, que nous présentons dans le Tableau 2: pour chacune des 70 personnes qui ont donné lieu à la création d'un onomastisme, nous indiquons (quand nous le savons) le nom, le prénom, les dates de naissance et de décès, le cas échéant, la nationalité et la discipline scientifique qui fut la sienne (dans la colonne DATES, une année entre parenthèses est celle d'une publication de l'auteur, quand nous ne disposions pas d'autres données chronologiques). Ces données, mêmes fragmentaires, laissent voir quelques faits intéressants.

D'abord une grande variété sur les plans chronologique et géographique: on y retrouve des Grecs de l'Antiquité, un Arabe du Moyen Âge, des Européens de toutes nationalités des quatre derniers siècles, des Américains à partir du tournant du $\mathrm{XX}^{\mathrm{e}}$ siècle. Les époques correspondent à la période culminante de chacune de ces quatre civilisations, ce qui bien sûr n'est pas un hasard. 
La colonne DISCIPLINE permet de constater d'où vient l'intelligence artificielle et, par le fait même, une bonne partie de sa terminologie: principalement des mathématiques et de la logique, cette dernière surtout à partir du $\mathrm{XIX}^{\mathrm{e}}$ siècle. On peut observer une certaine spécialisation avec les siècles: de la philosophie antique s'est dégagée la science mathématique, dont la logique constitue elle-même une branche de plus en plus autonome. D'autres disciplines sont représentées: la linguistique, la psychologie, la physique, etc. Se trouvent sûrement quelques informaticiens (sans parler des purs "cogniticiens») parmi les contemporains pour lesquels nous n'avons pas de données.

$\mathrm{Au}$ moins deux femmes ont mérité l'honneur éponymique. D'abord la mathématicienne Ada Byron, comtesse de Lovelace et fille de Lord Byron: son prénom a été donné au langage informatique Ada. Une autre femme, la logicienne Ruth Marcus, Américaine née en 1921, est sans doute à l'origine (nous n'avons pu certifier le lien) du terme analyseur Marcus (et de son synonyme parseur Marcus). C'est peut-être son prénom qui est représenté dans cet autre terme, analyseur de Ruth, pour lequel nous n'avons pas de définition.

Si l'on revient au tableau, on constate que les trois dernières colonnes donnent respectivement, pour chaque personne, le nombre de termes où l'anthroponyme $\mathrm{X}$ demeure tel quel, comme nom propre (ex.: formule de Bayes), le nombre de termes où l'éponyme est dérivé (shannon, réseau bayésien), puis le total de ces deux nombres.

$\mathrm{Si}$ on classe les noms selon ce dernier total, en ordre décroissant (Tableau 3), c'est le logicien George Boole qui domine le palmarès avec 25 termes issus de son nom. Si on classe selon la colonne des dérivés (Tableau 4), Boole domine toujours. Ce qui est interessant dans ce dernier tableau, c'est que, sur les 16 premiers noms (ceux qui donnent lieu à un dérivé ou plus), au moins 13 sont ceux de savants nés avant le $\mathrm{XX}^{\mathrm{e}}$ siècle. Cela semble confirmer que le facteur temps (qui permet de décanter les contributions scientifiques fondamentales) joue un rôle important dans la dérivation ou la lexicalisation d'un nom propre. 
Comme on peut alors s'y attendre, si on classe les données selon la colonne $\mathrm{X}$ (Tableau 5), ce sont des savants plus contemporains qui occupent les strates supérieures. La dérivation onomastique, comme la canonisation, semble demander une longue période d'examen posthume de la valeur du candidat. Du moins, dans le rigoureux domaine de la science. Car dans le domaine de la critique littéraire, artistique ou de la politique par exemple, où la préoccupation terminologique est absente, il est plus courant de jouer avec le nom des vivants pour en faire des dérivés qui, souvent, ne survivront pas au personnage.

Le Tableau 6 isole les 7 anthroponymes pour lesquels nous avons à la fois des éponymes gardant le nom propre et des éponymes dérivés: par exemple de Andrei Markov vient le terme chaîne de Markov, mais aussi système markovien. De ce nombre, les 3 noms où la deuxième forme est majoritaire sont les 3 plus anciens. On constate que la datation est importante et qu'il y a peut-être eu un changement de construction $\mathrm{N}$ prép $\mathrm{X} \rightarrow \mathrm{N} X$-ien au fil des siècles.

Mais il ne faut pas considérer que les seuls facteurs temps ou notoriété pour expliquer la répartition des onomastismes de formes $\mathrm{N}$ de $\mathrm{X}$ ou bien $\mathrm{N} \mathrm{X}$-ien. Les lois de la phonétique et de la dérivation françaises font que certains anthroponymes se prêtent mal à la suffixation en -ien. Ceux qui ont étudié les dérivés des noms d'auteurs (par exemple Anker Teilgård Laugesen, 1974, p. 267 et Colette Monsarrat, 1974, p. 447) ont remarqué qu'il y a de grands absents parmi les noms d'écrivains (même en ne considérant que les noms «bien français») qui dérivent en adjectifs. Parfois l'adjectif existe, mais son euphonie reste à démontrer (rousseauiste). On peut «réaménager» le radical (jusqu'à imaginer un ancêtre latin: Descartes $\rightarrow$ Cartesius $\rightarrow$ cartésien; Giraudoux $\rightarrow$ giralducien ) pour faciliter la dérivation, mais l'effet obtenu n'est pas toujours heureux.

Si le Français Joseph Fourier (1768-1830), qui a laissé son nom à de nombreux termes mathématiques (série de Fourier, transformation de Fourier, etc.), n'a pas connu l'honneur de la dérivation (contrairement à certains de ses confrères mathématiciens de l'époque), c'est peut-être que l'allitération dans l'adjectif attendu "fouriérien choque l'oreille. Il semble que l'adjectif fouriériste passe déjà mieux, 
puisqu'il désigne les partisans du système philosophique et économique de Charles Fourier, contemporain de Joseph, sans lien de parenté.

Les partisans du linguiste Benveniste, s'ils succombaient à l'«ismisme» généralisé de notre époque (l'abus de la dérivation en -isme et en -iste), seraient-ils pris au sérieux en se proclamant benvenististes? Comment qualifier la musique d'Olivier Messiaen ? Musique "messiaenne, musique "messiaenienne, musique "messiaenique, etc. ?

La chose se complique plus souvent s'il faut dériver à partir d'anthroponymes étrangers, comme c'est souvent le cas en science. Des adjectifs existants comme bayésien et hamiltonien ne sont peut-être pas des modèles d'harmonie française. Quel adjectif tirer de Earley? de Turing? Les termes algorithme "earléien et machine "turingienne ne semblent pas prêts de remplacer algorithme de Earley et machine de Turing.

\section{Les onomastismes du point de vue de $\mathbf{N}$ (déterminé)}

Nous avons relevé, dans le corpus, quelques doublets que l'on peut considérer comme des synonymes concurrents: cercle d'Euler / cercle eulérien, réseau de Bayes / réseau bayésien, algèbre de Boole / algèbre booléenne.

La question de la concurrence entre les formes de type $\mathrm{N}$ de $\mathrm{X}$ (tournure analytique) et $\mathrm{N}$ X-ien (adjectif dérivé) a été abordée par Anker Teilgård Laugesen (1974) et Marek Gawełko (1977). Leurs études concernent les noms d'auteurs et leurs dérivés, tels qu'ils sont utilisés par les critiques littéraires notamment. Anker Teilgård Laugesen et Marek Gawełko tentent de dégager des critères de répartition entre les deux formes (par exemple entre le théâtre de Racine et le théâtre racinien). Il faut toutefois préciser que leurs exemples sont des unités de discours et non des unités terminologiques.

Anker Teilgård Laugesen constate une grande variabilité et voit dans ce phénomène de concurrence une question de goût personnel de l'encodeur. L'adjectif est possible surtout quand on analyse l'œuvre, la production, contrairement à la vie privée par exemple (les gants de 
Pascal et non les gants pascaliens). Parfois l'adjectif peut remplacer sur $\mathrm{X}$ plutôt que de X: études moliéresques pour études sur Molière.

À ces critères externes, Marek Gawełko (1977) ajoute (en insistant qu'ils n'indiquent que des tendances) quelques critères internes de distribution des deux formes: le caractère plus ou moins général, abstrait, de l'unité substantif déterminé + adjectif dérivé et surtout la fréquence du mot primitif. En effet, peut intervenir le souci stylistique de rompre la monotonie créée par la répétition de la tournure analytique.

Dans le cas des unités terminologiques, leur caractère figé devrait en principe limiter de telles variations stylistiques. Il faudrait vérifier s'il arrive que, dans un même texte scientifique, un rédacteur alterne, par exemple, entre réseau de Bayes et réseau bayésien. Ou s'il introduit une première fois la notion avec la tournure analytique, plus transparente, puis passe ensuite à la forme adjectivale (qui devient plus motivée pour le lecteur quand celui-ci vient de rencontrer le nom propre, source de la dérivation) pour s'y tenir tout au long de son texte. Si la tournure adjectivale est exclusivement employée, on peut supposer son statut terminologique bien établi, à la fois sur le plan de la notion et de la désignation. On peut aussi se demander si l'adjectif dérivé ne prend pas un sens particulier bien précis.

$\mathrm{Si}$, dans le corpus, on observe la distribution des deux tournures selon le substantif déterminé (Tableau 7), ou base, il est difficile de dégager une tendance. Si l'on ordonne les bases selon la colonne des dérivés (Tableau 8), les substantifs les plus déterminés sont autant de type «concret» (graphe, circuit, distance), qu'abstrait (logique, raisonnement, variable). En intelligence artificielle, les termes véritablement concrets sont d'ailleurs rares.

La comparaison des trois listes (le Tableau 9 classe les données selon la colonne $\mathrm{X}$ ) ne permet pas de dégager d'opposition particulière (par exemple une opposition entre des concepts abstraits et des réalités plus concrètes ou bien entre des concepts «objectifs» par rapport à des concepts plus «subjectifs», «personnels»; opposition notion générale/notion spécifique bien précise). 
La base la plus fréquente est algorithme avec 18 unités créées, toutes du type $\mathrm{N}$ de $\mathrm{X}$. Une quinzaine de bases (Tableau 10) se retrouvent dans des matrices des deux types (chaîne de Markov à côté de chaîne eulérienne; distribution de Boltzmann à côté de distribution gaussienne, espace de Hilbert à côté de espace euclidien, etc.). La distribution semble donc à première vue assez aléatoire et ne permet pas de tirer des conclusions valables quant à la prédominance, par exemple, de bases à contenu sémantique indifférencié ou, au contraire, déterminé, les deux types étant présents.

En conclusion, l'étude menée sur les noms propres et de leurs dérivés dans le vocabulaire de l'intelligence artificielle permet de constater que ces derniers entrent, pour la majeure partie, dans la composition de syntagmes lexicalisés, correspondant à des notions dont le contenu ne peut se résumer à la somme des éléments qui les composent. Elle nous apprend également que c'est à la construction prépositionnelle qu'a recours le plus fréquemment la langue dans ce domaine de spécialité pour former des unités lexicales comprenant un nom propre ou un dérivé de nom propre. Enfin, elle renseigne également sur la provenance et l'origine de la terminologie de l'intelligence artificielle, domaine qui se trouve au carrefour de plusieurs disciplines. On l'a constaté, sa terminologie serait empruntée en bonne partie à celle de la logique et à celle des mathématiques, et non à l'informatique, ce que l'on suppose souvent.

Monique C. Cormier et Jean Fontaine: Département de linguistique et de traduction, Université de Montréal, CP 6128, Succ. A, Montréal (Québec) H3C 2J7

\section{Références}

AUTHIER, Michel (1989). «La réfraction et l'"oubli" cartésien», Michel Serres, dir., Éléments d'histoire des sciences, Paris, Bordas, pp. 251273. 
BOULANGER, Jean-Claude (1986). Aspects de l'interdiction dans la lexicographie française contemporaine, coll. «Lexicographica», (Series maior, 13), Tübingen, Max Niemeyer Verlag, IX + 166 p.

BOULANGER, Jean-Claude et Dorothy NAKOS (1988). Le syntagme terminologique. Bibliographie sélective et analytique (1960-1988), Publication K-7, Québec, Centre international de recherche sur le bilinguisme, $81 \mathrm{p}$.

CORMIER, Monique C. et Louis-Paul RIOUX (1991). «Procédés de formation et matrices terminogéniques en terminologie des systèmes experts», Meta, XXXVI(1), pp. 248-268.

DE CHANTAL, René (1961). «Les noms propres et leurs dérivés», Chroniques de français, Nouvelle édition revue et corrigée, Ottawa, Éditions de l'Université d'Ottawa, pp. 173-193.

DUGAS, Jean-Yves (1982). «Le provignement dans les noms propres», Travaux de terminologie et de linguistique I, Coll. Études, recherches et documentation, Québec, Gouvernement du Québec, pp. 21-39.

(1986). «Modalités d'inclusion et de traitement des gentilés, des adjectifs toponymiques et des dérivés de noms de personnes dans des dictionnaires de langue québécois», La lexicographie québécoise. Bilan et perspectives. Actes du colloque de 1985, Collection «Langue française au Québec», $3^{\mathrm{e}}$ section, $\mathrm{n}^{\circ} 8$, Sainte-Foy (Québec), Presses de l'Université Laval, pp. 233-256.

GAWEŁKO, Marek (1977). «Sur la concurrence des types de Pascal / pascalien», Revue romane, XII(1), pp. 123-126.

(1977a). «Analyse typologique des adjectifs romans de ressemblance dérivés de noms de personne», Studi italiani di linguistica teorica ed applicata, VI(1-2), pp. 141-159.

LAPIERRE, André (1989). «Problématique des éponymes en français contemporain", Dieter Kremer, dir., Actes du XVIII Congrès international de linguistique et de philologie romanes, Université de 
Trèves, 1986, Tübingen, Max Niemeyer Verlag, tome IV, section VII (Onomastique), pp. 588-597.

LAUGESEN, Anker Teilgård (1974). «Mots dérivés de noms d'auteurs», Revue romane, IX(2), pp. 255-276.

LAZURE, Noël (1988). Vocabulaire de l'intelligence artificielle, Bulletin de terminologie 184, Ottawa, Secrétariat d'État du Canada / Ministre des Approvisionnements et Services Canada, 2 vol., XV + $1217 \mathrm{p}$.

LETHUILLIER, Jacques et Monique C. CORMIER (1990). "Terminologie anglais-français des systèmes experts et des sujets connexes», Meta, XXXV(4), pp. 748-759.

(1991). «Terminologie anglais-français des systèmes experts et des sujets connexes. Suite», Meta, XXXVI(2/3), pp. 490-497.

MONSARRAT, Colette (1974). "Variations sur des noms d'auteurs", Vie et langage, 269, août, pp. 444-447.

OTMAN, Gabriel (1991). Vocabulaire d'intelligence artificielle. Paris, EC2 / Centre de terminologie et de néologie, $V+133 p$.

PAVEL, Silvia (1989). Intelligence logicielle. Dictionnaire françaisanglais. Réseau international de néologie et de terminologie, Module canadien, Ottawa, Secrétariat d'État du Canada, XII + 503 p.

QUEMADA, Bernard (1978). "Technique et langage», Bertrand Gille, dir., Histoire des techniques, Encyclopédie de la Pléiade, Paris, Gallimard, pp. 1146-1240.

REY, Alain, dir. (1992). Dictionnaire historique de la langue française. Paris, Dictionnaires Le Robert, 2 vol., 2387 p.

SCHWEICKARD, Wolfgang (1989). «Le traitement des formations déonomastiques dans la lexicographie française», Dieter Kremer, dir., Actes du XVIII Congrès international de linguistique et de philologie 
romanes, Université de Trèves, 1986, Tübingen, Max Niemeyer Verlag, tome IV, section VI (Lexicologie et lexicographie), pp. 242-253.

VAN HOOF, Henri (1986). «Les éponymes médicaux: essai de classification», Meta, XXXI(1), pp. 59-84.

WINSTON, Patrick Henry (1988). Intelligenceartificielle, texte français d'Annie Danzart et Jean-MichelMoreau, Paris, Addison-Wesley Europe/ InterÉditions, $528 \mathrm{p}$. 


\section{ANNEXES}

\section{TABLEAU 1 \\ MATRICES MORPHOSYNTAXIQUES}

$X=$ nom propre

$x, x$-isation, $x$-ien, etc. $=$ dérivé de nom propre $(x+$ suffixe $)$

$\mathrm{n}, \mathrm{adj}, \mathrm{adv}=$ catégories grammaticales

de, du, des = les prépositions de, du, des

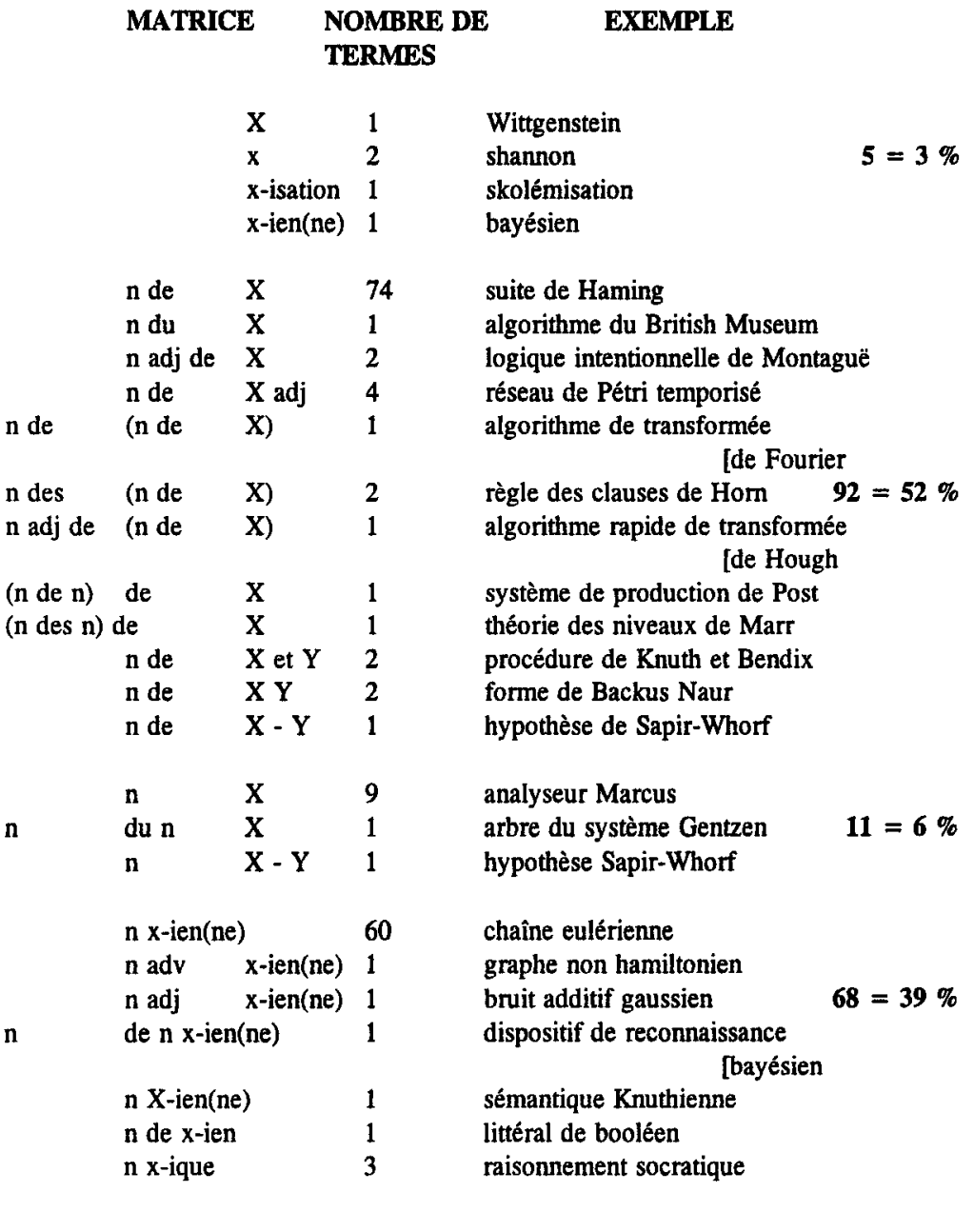


TABLEAU 2

DONNÉES BIOGRAPHIQUES DES PERSONNES QUI ONT DONNÉ LIEU

Ȧ LA CRÉATION D'UN ONOMASTISME

CLASSÉES DANS L'ORDRE ALPHABÉTIQUE DE NOMS

$\mathrm{X}=$ nombre de termes où l'anthroponyme apparait sous forme de nom propre

$\mathrm{D}=$ nombre de termes ou l'anthroponyme apparaitt sous forme de dérivé

Total $=$ total de $X+D$

\begin{tabular}{|c|c|c|c|c|c|c|c|}
\hline NOM & PRÉNOM & DATES & NATIONALITÉ & DISCIPLINE & $\mathbf{x}$ & $\mathbf{D}$ & TOTAL \\
\hline Byron Lovelace & Lady Ada & $1815-1851$ & anglaise & maths & 1 & & 1 \\
\hline Al Huwarizmi & Abdallah & $780-850$ & arabe & maths, astronomie & & 1 & 1 \\
\hline Aristote & & $-384--322$ & grecque & philo, logique & & 2 & 2 \\
\hline Backus & J. & (1978) & & & 2 & & 2 \\
\hline Bayes & Thomas & $1702-1761$ & anglaise & maths & 5 & 12 & 17 \\
\hline Bellman & Richard & $1920-$ & américaine & maths, rech. opérationnelle & 1 & & 1 \\
\hline Bendix & & & & & 2 & & 2 \\
\hline Boltzmann & Ludwig & $1844-1906$ & autrichienne & physique, philo des sciences & 2 & & 2 \\
\hline Boole & George & $1815-1864$ & anglaise & maths, logique & 1 & 24 & 25 \\
\hline Cherton & & & & & 1 & & 1 \\
\hline Chomsky & Avram Noam & $1928-$ & américaine & linguistique & 1 & & 1 \\
\hline Dempster & A. $\mathbf{P}$. & (1967) & & & 1 & & 1 \\
\hline Dijkstra & E. W. & (1959) & & & 1 & & 1 \\
\hline Diophante & & -200 & grecque & maths & & 1 & 1 \\
\hline Earley & J. & $(1968)$ & & & 3 & & 3 \\
\hline
\end{tabular}


TABLEAU 2 (suite)

\begin{tabular}{|c|c|c|c|c|c|c|c|}
\hline NOM & PRÉNOM & DATES & NATIONALITÉ & DISCIPLINE & $\mathbf{x}$ & $\mathbf{D}$ & TOTAL \\
\hline Eisenberg & & & & & 1 & & 1 \\
\hline Euclide & & -300 & grecque & maths & & 3 & 3 \\
\hline Euler & Leonhard & $1707-1783$ & suisse & maths & 1 & 8 & 9 \\
\hline Florès & F. & (1986) & & & 1 & & 1 \\
\hline Fourier & Joseph & $1768-1830$ & francaise & maths, physique & 2 & & 2 \\
\hline Gauss & Carl Friedrich & $1777-1855$ & allemande & astronomie, maths, physique & & 5 & 5 \\
\hline Gentzen & Gerhard & $1909-1945$ & allemande & logique & 1 & & 1 \\
\hline Golay & & & & & 1 & & 1 \\
\hline Grundy & & & & & 1 & & 1 \\
\hline Hamilton & William Rowan & $1805-1865$ & irlandaise & maths, physique & & 7 & 7 \\
\hline Haming & & & & & 1 & & 1 \\
\hline Hebb & Donald 0 . & $1904-$ & canadienne & psychologie & 1 & & 1 \\
\hline Herbrand & Jacques & $1908-1931$ & française & logique, maths & 6 & & 6 \\
\hline Hilbert & David & $1862-1943$ & allemande & maths & 4 & & 4 \\
\hline Hintikka & Jaakko & $1929-$ & finlandaise & logique, philo & 1 & & 1 \\
\hline Hoare & & & & & 2 & & 2 \\
\hline Hopfield & John Jos. & $1891-1953$ & & physique & 1 & & 1 \\
\hline Horn & Berthold & (1984) & & & 5 & & 5 \\
\hline Hough & P.V.C. & (1962) & & & 1 & & 1 \\
\hline Huffman & D. & (1971) & & & 2 & & 2 \\
\hline Kessels & & & & & 1 & & 1 \\
\hline Knuth & & & & & 3 & 1 & 4 \\
\hline Kripke & Saul & $1940-$ & américaine & logique & 1 & & 1 \\
\hline Kruskal & Martin D. & 1925- & américaine & physique, maths & 1 & & 1 \\
\hline
\end{tabular}




\section{TABLEAU 2 (suite)}

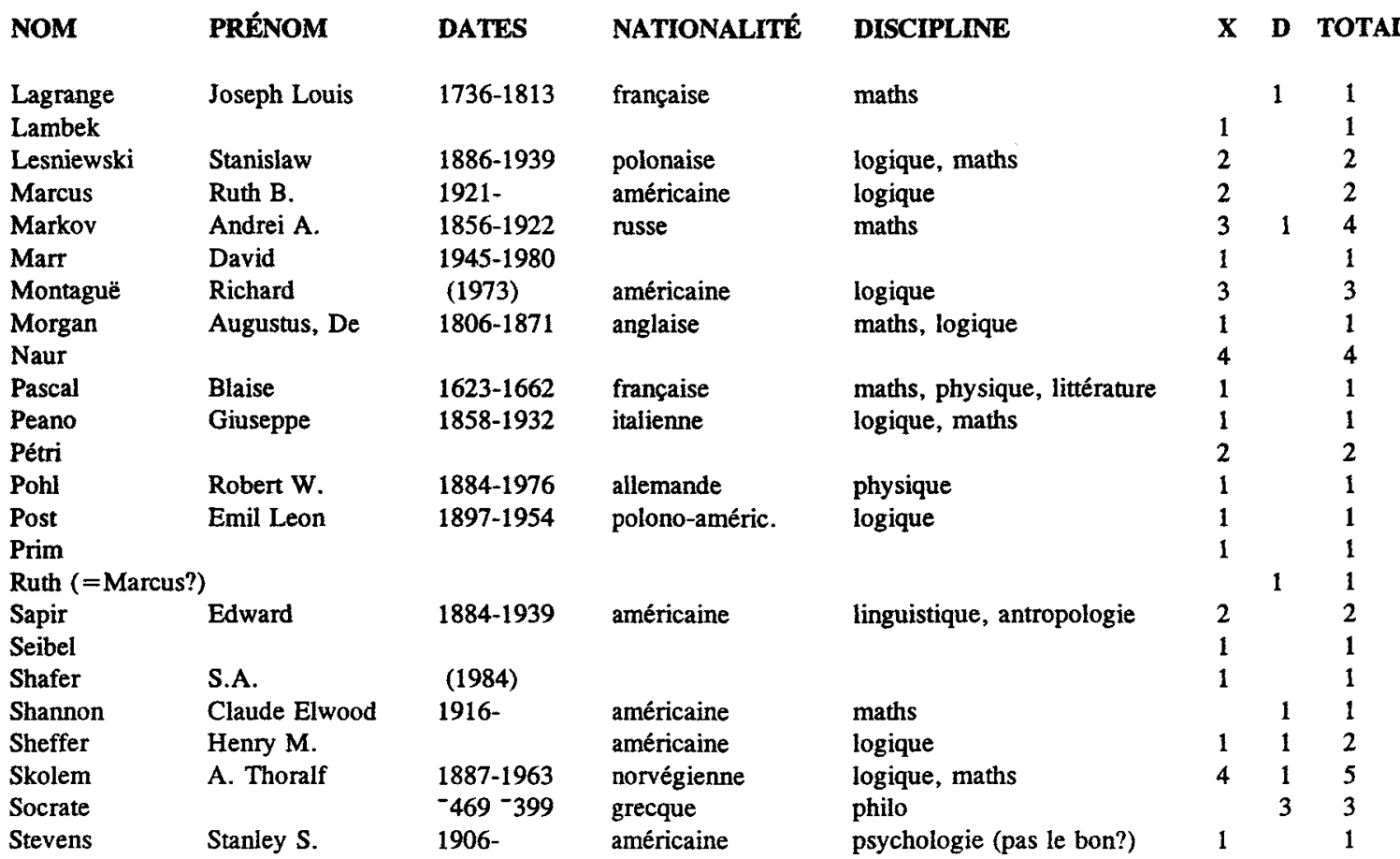


TABLEAU 2 (suite)

$\begin{array}{llcllll}\text { NOM } & \text { PRÉNOM } & \text { DATES } & \text { NATIONALITÉ } & \text { DISCIPLINE } & \text { X } & \text { D } \\ & & & & & 1 & 1 \\ \text { Tarski } & \text { Alfred } & 1902-1983 & \text { polono-améric. } & \text { logique, maths } & 3 & 3 \\ \text { Turing } & \text { Alan Mathison } & 1912-1954 & \text { anglaise } & \text { génie, maths } & 1 & 1 \\ \text { Venn } & \text { John } & 1834-1923 & \text { anglaise } & \text { logique } & 1 & 1 \\ \text { Viterbi } & \text { A.J. } & (1967) & & & 2 & 2 \\ \text { Whorf } & \text { Benjamin L. } & 1897-1941 & \text { américaine } & \text { linguistique } & 1 & 1 \\ \text { Winston } & \text { Patrick } & (1984) & & & 1 & 1 \\ \text { Wittgenstein } & \text { Ludwig } & 1889-1951 & \text { britannique d'origine logique, philo } & & & \end{array}$


TABLEAU 3

MÊMES DONNÉES QU'AU TABLEAU 2, MAIS CLASSÉES SELON LA COLONNE TOTAL EN ORDRE DÉCROISSANT

$\begin{array}{llllllll}\text { NOM } & \text { PRÉNOM } & \text { DATES } & \text { NATIONALITÉ } & \text { DISCIPLINE } & \text { X } & \text { DOTAL } \\ \text { Boole } & \text { George } & 1815-1864 & \text { anglaise } & \text { maths, logique } & 1 & 24 & 25 \\ \text { Bayes } & \text { Thomas } & 1702-1761 & \text { anglaise } & \text { maths } & \text { maths } & \mathbf{5} & 17 \\ \text { Euler } & \text { Leonhard } & 1707-1783 & \text { suisse } & \text { maths, physique } & \mathbf{8} & 9 \\ \text { Hamilton } & \text { William Rowan } & 1805-1865 & \text { irlandaise } & \text { logique, maths } & 7 & 7 \\ \text { Herbrand } & \text { Jacques } & 1908-1931 & \text { française } & \text { logique, maths } & 6 & 6 \\ \text { Skolem } & \text { A. Thoralf } & 1887-1963 & \text { norvégienne } & & 4 & 1 \\ \text { Horn } & \text { Berthold } & (1984) & & & 5 & 5 \\ \text { Gauss } & \text { Carl Friedrich } & 1777-1855 & \text { allemande } & \text { astronomie, maths, physique } & 5 & 5 \\ \text { Knuth } & & & & & 3 & 1 & 4 \\ \text { Markov } & \text { Andrei A. } & 1856-1922 & \text { russe } & \text { maths } & 3 & 1 \\ \text { Hilbert } & \text { David } & 1862-1943 & \text { allemande } & \text { maths } & 4 & 4 \\ \text { Naur } & & & & & 4 & 4 \\ \text { Turing } & \text { Alan Mathison } & 1912-1954 & \text { anglaise } & \text { génie, maths } & 3 & 3 \\ \text { Montaguë } & \text { Richard } & (1973) & \text { américaine } & \text { logique } & 3 & 3 \\ \text { Socrate } & & -469--399 & \text { grecque } & \text { philo } & 3 & 3 \\ \text { Earley } & \text { J. } & (1968) & & & 3 & 3 \\ \text { Euclide } & & -300 & \text { grecque } & \text { maths } & 3 & 3 \\ \text { Huffman } & \text { D. } & (1971) & & & 2 & 2 \\ \text { Whorf } & \text { Benjamin L. } & 1897-1941 & \text { américaine } & \text { linguistique } & 2 & 2 \\ \text { Aristote } & & -384--322 & \text { grecque } & \text { philo, logique } & 2\end{array}$


TABLEAU 3 (suite)

\begin{tabular}{|c|c|c|c|c|c|c|c|}
\hline NOM & PRÉNOM & DATES & NATIONALITÉ & DISCIPLINE & $\mathbf{x}$ & D & TOTAL \\
\hline Fourier & Joseph & $1768-1830$ & française & maths, physique & 2 & & 2 \\
\hline Marcus & Ruth B. & $1921-$ & américaine & logique & 2 & & 2 \\
\hline Pétri & & & & & 2 & & 2 \\
\hline Boltzmann & Ludwig & $1844-1906$ & autrichienne & physique, philo des sciences & 2 & & 2 \\
\hline Bendix & & & & & 2 & & 2 \\
\hline Hoare & & & & & 2 & & 2 \\
\hline Sheffer & Henry $M$. & & américaine & logique & 1 & 1 & 2 \\
\hline Sapir & Edward & $1884-1939$ & américaine & linguistique, antropologie & 2 & & 2 \\
\hline Backus & J. & (1978) & & & 2 & & 2 \\
\hline Lesniewski & Stanislaw & $1886-1939$ & polonaise & logique, maths & 2 & & 2 \\
\hline Wittgenstein & Ludwig & $1889-1951$ & $\begin{array}{l}\text { britannique d'origine } \\
\text { autrichienne }\end{array}$ & logique, philo & 1 & & 1 \\
\hline Stevens & Stanley S. & 1906- & américaine & psychologie (pas le bon?) & 1 & & 1 \\
\hline Golay & & & & & 1 & & 1 \\
\hline Hough & P.V.C. & (1962) & & & 1 & & 1 \\
\hline Gentzen & Gerhard & $1909-1945$ & allemande & logique & 1 & & 1 \\
\hline Hopfield & John Jos. & $1891-1953$ & & physique & 1 & & 1 \\
\hline Haming & & & & & 1 & & 1 \\
\hline Lagrange & Joseph Louis & $1736-1813$ & française & maths, astronomie & & 1 & 1 \\
\hline Peano & Giuseppe & $1858-1932$ & italienne & logique, maths & 1 & & 1 \\
\hline Florès & F. & $(1986)$ & & & 1 & & 1 \\
\hline Venn & John & $1834-1923$ & anglaise & logique & 1 & & 1 \\
\hline Eisenberg & & & & & 1 & & 1 \\
\hline
\end{tabular}


TABLEAU 3 (suite)

\begin{tabular}{|c|c|c|c|c|}
\hline NOM & PRÉNOM & DATES & NATIONALITÉ & DISCIPLINE \\
\hline \multicolumn{5}{|c|}{ Ruth (=Marcus?) } \\
\hline Bellman & Richard & $1920-$ & américaine & maths, rech. opérationnelle \\
\hline Post & Emil Leon & $1897-1954$ & polono-améric. & logique \\
\hline Hintikka & Jaakko & 1929- & finlandaise & logique, philo \\
\hline Shannon & Claude Elwood & 1916- & américaine & maths \\
\hline Hebb & Donald 0. & $1904-$ & canadienne & psychologie \\
\hline Shafer & S.A. & (1984) & & \\
\hline \multicolumn{5}{|l|}{ Grundy } \\
\hline Chomsky & Avram Noam & $1928-$ & américaine & linguistique \\
\hline $\begin{array}{l}\text { Diophante } \\
\text { Winston }\end{array}$ & Patrick & $\begin{array}{l}-200 \\
(1984)\end{array}$ & grecque & maths \\
\hline $\begin{array}{l}\text { Kruskal } \\
\text { Viterbi }\end{array}$ & $\begin{array}{l}\text { Martin D. } \\
\text { A.J. }\end{array}$ & $\begin{array}{l}1925- \\
(1967)\end{array}$ & américaine & physique, maths \\
\hline \multicolumn{5}{|l|}{ Prim } \\
\hline Dijkstra & E. W. & (1959) & & \\
\hline Pohl & Robert W. & $1884-1976$ & allemande & physique \\
\hline $\begin{array}{l}\text { Al Huwarizmi } \\
\text { Lambek }\end{array}$ & Abdallah & $780-850$ & arabe & maths, astronomie \\
\hline Seibel & & & & \\
\hline $\begin{array}{l}\text { Tarski } \\
\text { Cherton }\end{array}$ & Alfred & $1902-1983$ & polono-améric. & logique, maths \\
\hline Marr & David & $1945-1980$ & & \\
\hline Dempster & A. P. & $(1967)$ & & \\
\hline Kripke & Saul & $1940-$ & américaine & logique \\
\hline
\end{tabular}

X D TOTAL

$\begin{array}{lll} & 1 & 1 \\ 1 & & 1 \\ 1 & & 1 \\ 1 & & 1 \\ & 1 & 1 \\ 1 & & 1 \\ 1 & & 1 \\ 1 & & 1 \\ 1 & & 1 \\ & 1 & 1 \\ 1 & & 1 \\ 1 & & 1 \\ 1 & & 1 \\ 1 & & 1 \\ 1 & & 1 \\ 1 & & 1 \\ & 1 & 1 \\ 1 & & 1 \\ 1 & & 1 \\ 1 & & 1 \\ 1 & & 1 \\ 1 & & 1 \\ 1 & & 1 \\ 1 & & 1 \\ & & \end{array}$


TABLEAU 3 (suite)

$\begin{array}{lllllll}\text { NOM } & \text { PRÉNOM } & \text { DATES } & \text { NATIONALITÉ } & \text { DISCIPLINE } & \text { X } & \text { TOTAL } \\ \text { Byron Lovelace } & \text { Lady Ada } & 1815-1851 & \text { anglaise } & \text { maths } & 1 & 1 \\ \text { Pascal } & \text { Blaise } & 1623-1662 & \text { française } & \text { maths, physique, littérature } & 1 & 1 \\ \text { Morgan } & \text { Augustus, De } & 1806-1871 & \text { anglaise } & \text { maths, logique } & 1 & 1 \\ \text { Kessels } & & & & & 1 & 1\end{array}$


TABLEAU 4

MÊMES DONNÉES QƯ'AU TABLEAU 2, MAIS CLASSÉES SELON LA COLONNE D

\begin{tabular}{|c|c|c|c|c|c|c|c|}
\hline NOM & PRÉNOM & DATES & NATIONALITÉ & DISCIPLINE & $\mathbf{X}$ & $\mathbf{D}$ & TOTAL \\
\hline Boole & George & $1815-1864$ & anglaise & maths, logique & 1 & 24 & 25 \\
\hline Bayes & Thomas & $1702-1761$ & anglaise & maths & 5 & 12 & 17 \\
\hline Euler & Leonhard & $1707-1783$ & suisse & maths & 1 & 8 & 9 \\
\hline Hamilton & William Rowan & $1805-1865$ & irlandaise & maths, physique & & 7 & 7 \\
\hline Gauss & Carl Friedrich & $1777-1855$ & allemande & astronomie, maths, physique & & 5 & 5 \\
\hline Euclide & & -300 & grecque & maths & & 3 & 3 \\
\hline Socrate & & $-469--399$ & grecque & philo & & 3 & 3 \\
\hline Aristote & & $-384--322$ & grecque & philo, logique & & 2 & 2 \\
\hline Knuth & & & & & 3 & 1 & 4 \\
\hline Markov & Andrei $\mathbf{A}$. & $1856-1922$ & russe & maths & 3 & 1 & 4 \\
\hline Lagrange & Joseph Louis & $1736-1813$ & française & maths, astronomie & & 1 & 1 \\
\hline Skolem & A. Thoralf & $1887-1963$ & norvégienne & logique, maths & 4 & 1 & 5 \\
\hline Al Huwarizmi & Abdallah & $780-850$ & arabe & maths, astronomie & & 1 & 1 \\
\hline Shannon & Claude Elwood & $1916-$ & américaine & maths & & 1 & 1 \\
\hline Sheffer & Henry $\mathbf{M}$ & & américaine & logique & 1 & 1 & 2 \\
\hline Diophante & & -200 & grecque & maths & & 1 & 1 \\
\hline Herbrand & Jacques & $1908-1931$ & française & logique, maths & 6 & & 6 \\
\hline Hilbert & David & $1862-1943$ & allemande & maths & 4 & & 4 \\
\hline Hopfield & John Jos. & $1891-1953$ & & physique & 1 & & 1 \\
\hline Hebb & Donald $\mathbf{O}$. & 1904- & canadienne & psychologie & 1 & & 1 \\
\hline
\end{tabular}


TABLEAU 4 (suite)

\begin{tabular}{|c|c|c|c|c|c|c|c|}
\hline NOM & PRÉNOM & DATES & NATIONALITÉ & DISCIPLINE & $\mathbf{X}$ & $\mathbf{D}$ & TOTAL \\
\hline Sapir & Edward & $1884-1939$ & américaine & linguistique, antropologie & 2 & & 2 \\
\hline Grundy & & & & & 1 & & 1 \\
\hline Cherton & & & & & 1 & & 1 \\
\hline Hoare & & & & & 2 & & 2 \\
\hline Hough & P.V.C. & (1962) & & & 1 & & 1 \\
\hline Fourier & Joseph & $1768-1830$ & française & maths, physique & 2 & & 2 \\
\hline Golay & & & & & 1 & & 1 \\
\hline Huffman & D. & (1971) & & & 2 & & 2 \\
\hline Dempster & A. P. & (1967) & & & 1 & & 1 \\
\hline Earley & J. & (1968) & & & 3 & & 3 \\
\hline Prim & & & & & 1 & & 1 \\
\hline Gentzen & Gerhard & $1909-1945$ & allemande & logique & 1 & & 1 \\
\hline Horn & Berthold & (1984) & & & 5 & & 5 \\
\hline Chomsky & Avram Noam & $1928-$ & américaine & linguistique & 1 & & 1 \\
\hline Pascal & Blaise & $1623-1662$ & française & maths, physique, littérature & 1 & & 1 \\
\hline Post & Emil Leon & $1897-1954$ & polono-améric. & logique & 1 & & 1 \\
\hline Kripke & Saul & $1940-$ & américaine & logique & 1 & & 1 \\
\hline Bellman & Richard & 1920 & américaine & maths, rech. opérationnelle & 1 & & 1 \\
\hline Haming & & & & & 1 & & 1 \\
\hline Hintikka & Jaakko & 1929 - & finlandaise & logique, philo & 1 & & 1 \\
\hline Bendix & & & & & 2 & & 2 \\
\hline Florès & F. & (1986) & & & 1 & & 1 \\
\hline Shafer & S.A. & (1984) & & & 1 & & 1 \\
\hline Eisenberg & & & & & 1 & & 1 \\
\hline
\end{tabular}


TABLEAU 4 (suite)

\begin{tabular}{|c|c|c|c|c|c|c|c|}
\hline NOM & PRÉNOM & DATES & NATIONALITÉ & DISCIPLINE & $\mathbf{x}$ & $\mathbf{D}$ & TOTAL \\
\hline Tarski & Alfred & $1902-1983$ & polono-améric. & logique, maths & 1 & & 1 \\
\hline Viterbi & A.J. & $(1967)$ & & & 1 & & 1 \\
\hline Wittgenstein & Ludwig & $1889-1951$ & $\begin{array}{l}\text { britannique d'origine } \\
\text { autrichienne }\end{array}$ & logique, philo & 1 & & 1 \\
\hline Backus & $\mathrm{J}$ & (1978) & & & 2 & & 2 \\
\hline Whorf & Benjamin L. & $1897-1941$ & américaine & linguistique & 2 & & 2 \\
\hline Stevens & Stanley $S$. & $1906-$ & américaine & psychologie (pas le bon?) & 1 & & 1 \\
\hline Dijkstra & E. W. & (1959) & & & 1 & & 1 \\
\hline Marcus & Ruth B. & $1921-$ & américaine & logique & 2 & & 2 \\
\hline Ruth (= Marcus? & & & & & & 1 & 1 \\
\hline Lambek & & & & & 1 & & 1 \\
\hline Boltzmann & Ludwig & $1844-1906$ & autrichienne & physique, philo des sciences & 2 & & 2 \\
\hline Turing & Alan Mathison & $1912-1954$ & anglaise & génie, maths & 3 & & 3 \\
\hline Lesniewski & Stanislaw & $1886-1939$ & polonaise & logique, maths & 2 & & 2 \\
\hline Byron Lovelace & Lady Ada & $1815-1851$ & anglaise & maths & 1 & & 1 \\
\hline Seibel & & & & & $i$ & & 1 \\
\hline Pohl & Robert W. & $1884-1976$ & allemande & physique & $i$ & & 1 \\
\hline Montaguë & Richard & (1973) & américaine & logique & 3 & & 3 \\
\hline Venn & John & $1834-1923$ & anglaise & logique & 1 & & 1 \\
\hline Winston & Patrick & (1984) & & & 1 & & 1 \\
\hline Kruskal & Martin D. & 1925 & américaine & physique, maths & 1 & & 1 \\
\hline Peano & Giuseppe & $1858-1932$ & italienne & logique, maths & 1 & & 1 \\
\hline Marr & David & $1945-1980$ & & & 1 & & 1 \\
\hline Naur & & & & & 4 & & 4 \\
\hline
\end{tabular}


TABLEAU 4 (suite)

NOM

Morgan

Pétri

Kessels

\section{PRÉNOM}

Augustus, De

DATES

1806-1871

NATIONALITÉ

anglaise

DISCIPLINE

maths, logique
X D

1

2

TOTAL

1 
TABLEAU 5

MÊMES DONNÉES QU'AU TABLEAU 2,

MAIS CLASSÉES SELON LA COLONNE X

\begin{tabular}{|c|c|c|c|c|c|c|c|}
\hline NOM & PRÉNOM & DATES & NATIONALITÉ & DISCIPLINE & $\mathbf{x}$ & D & TOTAL \\
\hline Herbrand & Jacques & $1908-1931$ & française & logique, maths & 6 & & 6 \\
\hline Bayes & Thomas & $1702-1761$ & anglaise & maths & 5 & 12 & 17 \\
\hline Horn & Berthold & (1984) & & & 5 & & 5 \\
\hline Naur & & & & & 4 & & 4 \\
\hline Skolem & A. Thoralf & $1887-1963$ & norvégienne & logique, maths & 4 & 1 & 5 \\
\hline Hilbert & David & $1862-1943$ & allemande & maths & 4 & & 4 \\
\hline Knuth & & & & & 3 & 1 & 4 \\
\hline Turing & Alan Mathison & $1912-1954$ & anglaise & génie, maths & 3 & & 3 \\
\hline Earley & J. & (1968) & & & 3 & & 3 \\
\hline Markov & Andrei $\mathrm{A}$. & $1856-1922$ & russe & maths & 3 & 1 & 4 \\
\hline Montaguë & Richard & (1973) & américaine & logique & 3 & & 3 \\
\hline Fourier & Joseph & $1768-1830$ & française & maths, physique & 2 & & 2 \\
\hline Huffman & D. & $(1971)$ & & & 2 & & 2 \\
\hline Pétri & & & & & 2 & & 2 \\
\hline Backus & J. & (1978) & & & 2 & & 2 \\
\hline Hoare & & & & & 2 & & 2 \\
\hline Lesniewski & Stanislaw & $1886-1939$ & polonaise & logique, maths & 2 & & 2 \\
\hline Sapir & Edward & $1884-1939$ & américaine & linguistique, antropologie & 2 & & 2 \\
\hline Whorf & Benjamin L. & 1897-1941 & américaine & linguistique & 2 & & 2 \\
\hline Bendix & & & & & 2 & & 2 \\
\hline
\end{tabular}


TABLEAU 5 (suite)

\begin{tabular}{|c|c|c|c|c|c|c|c|}
\hline NOM & PRÉNOM & DATES & NATIONALITÉ & DISCIPLINE & $\mathbf{x}$ & $\mathbf{D}$ & TOTAL \\
\hline Boltzmann & Ludwig & 1844-1906 & autrichienne & physique, philo des sciences & 2 & & 2 \\
\hline Marcus & Ruth B. & $1921-$ & américaine & logique & 2 & & 2 \\
\hline Boole & George & $1815-1864$ & anglaise & maths, logique & 1 & 24 & 25 \\
\hline Haming & & & & & 1 & & 1 \\
\hline Stevens & Stanley S. & 1906- & américaine & psychologie (pas le bon?) & 1 & & 1 \\
\hline Gentzen & Gerhard & 1909-1945 & allemande & logique & 1 & & 1 \\
\hline Shafer & S.A. & (1984) & & & 1 & & 1 \\
\hline Florès & F. & (1986) & & & 1 & & 1 \\
\hline Hopfield & John Jos. & $1891-1953$ & & physique & 1 & & 1 \\
\hline Hough & P.V.C. & $(1962)$ & & & 1 & & 1 \\
\hline Prim & & & & & 1 & & 1 \\
\hline Golay & & & & & 1 & & 1 \\
\hline Kripke & Saul & 1940 & américaine & logique & 1 & & 1 \\
\hline Kruskal & Martin D. & $1925-$ & américaine & physique, maths & 1 & & $i$ \\
\hline Hintikka & Jaakko & $1929-$ & finlandaise & logique, philo & 1 & & 1 \\
\hline Wittgenstein & Ludwig & $1889-1951$ & $\begin{array}{l}\text { britannique d'origine } \\
\text { autrichienne }\end{array}$ & logique, philo & 1 & & 1 \\
\hline Bellman & Richard & $1920-$ & américaine & maths, rech. opérationnelle & 1 & & 1 \\
\hline Eisenberg & & & & & 1 & & 1 \\
\hline Winston & Patrick & (1984) & & & 1 & & 1 \\
\hline Venn & John & $1834-1923$ & anglaise & logique & 1 & & 1 \\
\hline Cherton & & & & & 1 & & 1 \\
\hline \multicolumn{2}{|c|}{ Ruth (=Marcus?) } & & & & & 1 & 1 \\
\hline \multicolumn{2}{|l|}{ Seibel } & & & & 1 & & 1 \\
\hline
\end{tabular}


TABLEAU 5 (suite)

\begin{tabular}{|c|c|c|c|c|c|c|c|}
\hline NOM & PRÉNOM & DATES & NATIONALITÉ & DISCIPLINE & $\mathbf{x}$ & $\mathbf{D}$ & TOTAL \\
\hline Hebb & Donald $O$. & 1904- & canadienne & psychologie & 1 & & 1 \\
\hline Viterbi & A.J. & $(1967)$ & & & 1 & & 1 \\
\hline Sheffer & Henry $\mathrm{M}$. & & américaine & logique & 1 & 1 & 2 \\
\hline Pohl & Robert W. . & $1884-1976$ & allemande & physique & 1 & & 1 \\
\hline Lambek & & & & & 1 & & 1 \\
\hline Euler & Leonhard & $1707-1783$ & suisse & maths & 1 & 8 & 9 \\
\hline Chomsky & Avram Noam & $1928-$ & américaine & linguistique & 1 & & 1 \\
\hline Tarski & Alfred & $1902-1983$ & polono-améric. & logique, maths & 1 & & 1 \\
\hline Kessels & & & & & 1 & & 1 \\
\hline Pascal & Blaise & $1623-1662$ & française & maths, physique, littérature & 1 & & 1 \\
\hline Peano & Giuseppe & $1858-1932$ & italienne & logique, maths & 1 & & 1 \\
\hline Morgan & Augustus, De & $1806-1871$ & anglaise & maths, logique & 1 & & 1 \\
\hline Dijkstra & E. W. & (1959) & & & 1 & & 1 \\
\hline Marr & David & $1945-1980$ & & & 1 & & 1 \\
\hline Byron Lovelace & Lady Ada & $1815-1851$ & anglaise & maths & 1 & & 1 \\
\hline Post & Emil Leon & $1897-1954$ & polono-améric. & logique & 1 & & 1 \\
\hline Grundy & & & & & 1 & & 1 \\
\hline Dempster & A. P. & (1967) & & & 1 & & 1 \\
\hline Al Huwarizmi & Abdallah & $780-850$ & arabe & maths, astronomie & & 1 & 1 \\
\hline Shannon & Claude Elwood & $1916-$ & américaine & maths & & 1 & 1 \\
\hline Hamilton & William Rowan & $1805-1865$ & irlandaise & maths, physique & & 7 & 7 \\
\hline Euclide & & -300 & grecque & maths & & 3 & 3 \\
\hline Diophante & & -200 & grecque & maths & & 1 & 1 \\
\hline Aristote & & $-384--322$ & grecque & philo, logique & & 2 & 2 \\
\hline
\end{tabular}


TABLEAU 5 (suite)

\begin{tabular}{|c|c|c|c|c|c|c|c|}
\hline NOM & PRÉNOM & DATES & NATIONALITÉ & DISCIPLINE & $\mathbf{x}$ & $\mathbf{D}$ & TOTAL \\
\hline Gauss & Carl Friedrich & $1777-1855$ & allemande & astronomie, maths, physique & & 5 & 5 \\
\hline Lagrange & Joseph Louis & $1736-1813$ & française & maths, astronomie & & 1 & 1 \\
\hline Socrate & & $-469--399$ & grecque & philo & & 3 & 3 \\
\hline
\end{tabular}


TABLEAU 6

NOMS APPARAISSANT SOUS LES FORMES X ET D

\begin{tabular}{|c|c|c|c|c|c|c|c|}
\hline NOM & PRÉNOM & DATES & NATIONALITÉ & DISCIPLINE & $\mathbf{x}$ & D & TOTAL \\
\hline Bayes & Thomas & $1702-1761$ & anglaise & maths & 5 & 12 & 17 \\
\hline Boole & George & $1815-1864$ & anglaise & maths, logique & 1 & 24 & 25 \\
\hline Euler & Leonhard & $1707-1783$ & suisse & maths & 1 & 8 & 9 \\
\hline Knuth & & & & & 3 & 1 & 4 \\
\hline Markov & Andrei $\mathrm{A}$. & $1856-1922$ & russe & maths & 3 & 1 & 4 \\
\hline Sheffer & Henry $\mathrm{M}$. & & américaine & logique & 1 & 1 & 2 \\
\hline Skolem & A. Thoralf & $1887-1963$ & norvégienne & logique, maths & 4 & 1 & 5 \\
\hline
\end{tabular}


TABLEAU 7

LISTE ALPHABÉTIQUE DES BASES MODIFIÉES

PAR UN NOM PROPRE OU UN DÉRIVÉ

\begin{tabular}{|c|c|c|c|}
\hline NOM & $\mathbf{X}$ & $\mathbf{D}$ & TOTAL \\
\hline algorithme & 18 & & 18 \\
\hline algèbre & 1 & 1 & 2 \\
\hline analyseur & 3 & & 3 \\
\hline apprentissage & & 1 & 1 \\
\hline arbre & 1 & & 1 \\
\hline atome & 1 & & 1 \\
\hline attribut & & 1 & 1 \\
\hline barre & 1 & & 1 \\
\hline base & 1 & & 1 \\
\hline bruit additif & & 1 & 1 \\
\hline calcul & 1 & 1 & 2 \\
\hline caractérisation & & 1 & 1 \\
\hline cercle & 1 & 1 & 2 \\
\hline chaîne & 1 & 2 & 3 \\
\hline chemin & & 1 & 1 \\
\hline circuit & & 2 & 2 \\
\hline clause & 5 & & 5 \\
\hline compilateur & 1 & & 1 \\
\hline concept & & 1 & 1 \\
\hline conditionnement & 1 & & 1 \\
\hline conférence & 1 & & 1 \\
\hline
\end{tabular}

\begin{tabular}{|c|c|c|c|}
\hline NOM & $\mathbf{X}$ & D & TOTAI \\
\hline connecteur & & 1 & 1 \\
\hline constante & 1 & & 1 \\
\hline cycle & & 2 & 2 \\
\hline $\begin{array}{l}\text { diagramme } \\
\text { dispositif }\end{array}$ & 1 & & 1 \\
\hline de reconnaissance & & 1 & 1 \\
\hline distance & & 2 & 2 \\
\hline distribution & 1 & 1 & 2 \\
\hline équation & & 2 & 2 \\
\hline espace & 1 & 1 & 2 \\
\hline fait & & 1 & 1 \\
\hline filtrage & & 1 & 1 \\
\hline foncteur & & 1 & 1 \\
\hline fonction & 2 & & 2 \\
\hline formalisme & 1 & 1 & 2 \\
\hline forme & 2 & & 2 \\
\hline formule & 1 & & 1 \\
\hline grammaire & 2 & 1 & 3 \\
\hline graphe & & 3 & 3 \\
\hline géométrie & & 1 & 1 \\
\hline hypothèse & 2 & & 2 \\
\hline
\end{tabular}

$\begin{array}{lccc}\text { NOM } & \text { X } & \text { D } & \text { TOTA } \\ \text { ignorance } & & 1 & 1 \\ \text { image } & & 1 & 1 \\ \text { inférence } & & 1 & 1 \\ \text { littéral } & & 1 & 1 \\ \text { logique } & 5 & 2 & 7 \\ \text { loi } & 2 & & 2 \\ \text { machine } & 3 & & 3 \\ \text { matrice } & & 1 & 1 \\ \text { mode } & & 1 & 1 \\ \text { modélisation } & 1 & & 1 \\ \text { modèle } & 2 & 1 & 3 \\ \text { multigraphe } & & 1 & 1 \\ \text { mémoire } & 1 & & 1 \\ \text { méthode } & 1 & 1 & 2 \\ \text { notation } & 1 & & 1 \\ \text { opérateur } & & 1 & 1 \\ \text { opération } & & 1 & 1 \\ \text { paramètre } & & 1 & 1 \\ \text { parcours } & & 1 & 1 \\ \text { parseur } & 2 & & 2 \\ \text { probabilité } & & 1 & 1\end{array}$


TABLEAU 7 (suite)

\begin{tabular}{|c|c|c|c|c|c|c|c|}
\hline NOM & $\mathbf{x}$ & D & TOTAL & NOM & $\mathbf{X}$ & D & TOTAL \\
\hline problème & & 1 & 1 & type & & 1 & 1 \\
\hline procédure & 2 & 1 & 3 & tâche & 1 & & 1 \\
\hline raisonnement & & 2 & 2 & univers & 1 & & 1 \\
\hline raisonneur & & 1 & 1 & valeur & & 1 & 1 \\
\hline recherche & & 1 & 1 & variable & & 2 & 2 \\
\hline reconnaissance & & 1 & 1 & vecteur & & 1 & 1 \\
\hline relaxation & & 1 & 1 & voisinage & 1 & & 1 \\
\hline réseau & 4 & 1 & 5 & & & & \\
\hline règle & 2 & & 2 & & & & \\
\hline statistique & & 1 & 1 & & & & \\
\hline suite & 1 & & 1 & & & & \\
\hline symbole & 1 & & 1 & & & & \\
\hline syntaxe & 1 & & 1 & & & & \\
\hline système & 5 & 2 & 7 & & & & \\
\hline sémantique & 4 & 1 & 5 & & & & \\
\hline test & 1 & & 1 & & & & \\
\hline théorie & 2 & & 2 & & & & \\
\hline théorème & 3 & & 3 & & & & \\
\hline tour & 1 & & 1 & & & & \\
\hline transformation & 2 & & 2 & & & & \\
\hline transformée & 2 & & 2 & & & & \\
\hline treillis & & 1 & 1 & & & & \\
\hline tri & & 1 & 1 & & & & \\
\hline
\end{tabular}




\section{TABLEAU 8}

MÊMES DONNÉES QU'AU TABLEAU 7, MAIS CLASSÉES SELON LA COLONNE DÉRIVÉ

\begin{tabular}{|c|c|c|c|c|c|c|c|c|c|c|c|}
\hline NOM & $\mathbf{X}$ & $\mathbf{D}$ & TOTAL & NOM & $\mathbf{X}$ & $\mathbf{D}$ & TOTAL & NOM & $\mathbf{X}$ & $\mathbf{D}$ & To' \\
\hline graphe & & 3 & 3 & bruit additif & & 1 & 1 & géométrie & & 1 & 1 \\
\hline chaîne & 1 & 2 & 3 & formalisme & 1 & 1 & 2 & ignorance & & 1 & 1 \\
\hline cycle & & 2 & 2 & méthode & 1 & 1 & 2 & statistique & & 1 & 1 \\
\hline distance & & 2 & 2 & grammaire & 2 & 1 & 3 & raisonneur & & 1 & 1 \\
\hline circuit & & 2 & 2 & fait & & 1 & 1 & parcours & & 1 & 1 \\
\hline variable & & 2 & 2 & cercle & 1 & 1 & 2 & concept & & 1 & 1 \\
\hline équation & & 2 & 2 & apprentissage & & 1 & 1 & matrice & & 1 & 1 \\
\hline foncteur & & 1 & 1 & recherche & & 1 & 1 & probabilité & & 1 & 1 \\
\hline filtrage & & 1 & 1 & espace & 1 & 1 & 2 & opération & & 1 & 1 \\
\hline distribution & 1 & 1 & 2 & problème & & 1 & 1 & treillis & & 1 & 1 \\
\hline vecteur & & 1 & 1 & réseau & 4 & 1 & 5 & inférence & & 1 & 1 \\
\hline calcul & 1 & 1 & 2 & sémantique & 4 & 1 & 5 & type & & 1 & 1 \\
\hline caractérisation & & 1 & 1 & dispositif de & & & & fonction & 2 & & 2 \\
\hline mode & & 1 & 1 & reconnaissance & & 1 & 1 & formule & 1 & & 1 \\
\hline
\end{tabular}




\section{TABLEAU 8 (suite)}

\begin{tabular}{|c|c|c|}
\hline NOM & $\mathbf{X} \mathbf{D}$ & TO' \\
\hline règle & 2 & 2 \\
\hline hypothèse & 2 & \\
\hline théorie & 2 & \\
\hline base & 1 & 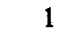 \\
\hline théorème & 3 & 3 \\
\hline clause & 5 & 5 \\
\hline algorithme & 18 & 18 \\
\hline suite & 1 & 1 \\
\hline symbole & 1 & 1 \\
\hline arbre & 1 & 1 \\
\hline forme & 2 & 2 \\
\hline univers & 1 & 1 \\
\hline barre & 1 & 1 \\
\hline notation & 1 & 1 \\
\hline loi & 2 & 2 \\
\hline atome & 1 & 1 \\
\hline machine & 3 & 3 \\
\hline analyseur & 3 & 3 \\
\hline syntaxe & 1 & 1 \\
\hline tâche & 1 & 1 \\
\hline transformation & 2 & 2 \\
\hline test & 1 & 1 \\
\hline conditionnement & 1 & 1 \\
\hline
\end{tabular}

\section{NOM \\ X D TOTAL}

$\begin{array}{lll}\text { conférence } & 1 & 1 \\ \text { constante } & 1 & 1 \\ \text { mémoire } & 1 & 1 \\ \text { diagramme } & 1 & 1 \\ \text { modélisation } & 1 & 1 \\ \text { tour } & 1 & 1 \\ \text { compilateur } & 1 & 1 \\ \text { transformée } & 2 & 2 \\ \text { parseur } & 2 & 2 \\ \text { voisinage } & 1 & 1\end{array}$


TABLEAU 9

MÊMES DONNÉES QU'AU TABLEAU 7,

MAIS CLASSÉES SELON LA COLONNE X

\begin{tabular}{|c|c|c|c|c|c|c|c|c|c|c|c|}
\hline NOM & $\mathbf{x}$ & D & TOTAL & NOM & $\mathbf{x}$ & $\mathbf{D}$ & TOTAL & NOM & $\mathbf{x}$ & D & TOTAL \\
\hline algorithme & 18 & & 18 & fonction & 2 & & 2 & notation & 1 & & 1 \\
\hline système & 5 & 2 & 7 & calcul & 1 & 1 & 2 & tâche & 1 & & 1 \\
\hline clause & 5 & & 5 & tour & 1 & & 1 & cercle & 1 & 1 & 2 \\
\hline logique & 5 & 2 & 7 & univers & 1 & & 1 & conditionnement & 1 & & 1 \\
\hline sémantique & 4 & 1 & 5 & diagramme & 1 & & 1 & symbole & 1 & & 1 \\
\hline réseau & 4 & 1 & 5 & formalisme & 1 & 1 & 2 & méthode & 1 & 1 & 2 \\
\hline analyseur & 3 & & 3 & voisinage & 1 & & 1 & chaîne & 1 & 2 & 3 \\
\hline théorème & 3 & & 3 & compilateur & 1 & & 1 & distribution & 1 & 1 & 2 \\
\hline machine & 3 & & 3 & constante & 1 & & 1 & modélisation & 1 & & 1 \\
\hline règle & 2 & & 2 & barre & 1 & & 1 & base & 1 & & 1 \\
\hline transformation & 2 & & 2 & algèbre & 1 & 1 & 2 & bruit additif & & 1 & 1 \\
\hline procédure & 2 & 1 & 3 & espace & 1 & 1 & 2 & type & & 1 & 1 \\
\hline forme & 2 & & 2 & atome & 1 & & 1 & connecteur & & 1 & 1 \\
\hline grammaire & 2 & 1 & 3 & mémoire & 1 & & 1 & littéral & & 1 & 1 \\
\hline transformée & 2 & & 2 & formule & 1 & & 1 & opérateur & & 1 & 1 \\
\hline parseur & 2 & & 2 & arbre & 1 & & 1 & image & & 1 & 1 \\
\hline théorie & 2 & & 2 & syntaxe & 1 & & 1 & caractérisation & & 1 & 1 \\
\hline hypothèse & 2 & & 2 & test & 1 & & 1 & problème & & 1 & 1 \\
\hline loi & 2 & & 2 & suite & 1 & & 1 & circuit & & 2 & 2 \\
\hline modèle & 2 & 1 & 3 & conférence & 1 & & 1 & concept & & 1 & 1 \\
\hline
\end{tabular}


TABLEAU 9 (suite)

\begin{tabular}{|c|c|c|}
\hline NOM & D & $\mathbf{T O}^{\prime}$ \\
\hline opération & 1 & 1 \\
\hline reconnaissance & 1 & 1 \\
\hline graphe & 3 & 3 \\
\hline fait & 1 & 1 \\
\hline relaxation & 1 & 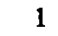 \\
\hline inférence & 1 & 1 \\
\hline statistique & 1 & 1 \\
\hline ignorance & 1 & 1 \\
\hline apprentissage & 1 & 1 \\
\hline valeur & 1 & 1 \\
\hline cycle & 2 & 2 \\
\hline vecteur & 1 & 1 \\
\hline géométrie & 1 & 1 \\
\hline attribut & 1 & 1 \\
\hline paramètre & 1 & $t$ \\
\hline recherche & 1 & 1 \\
\hline chemin & 1 & 1 \\
\hline raisonnement & 2 & 2 \\
\hline multigraphe & 1 & 1 \\
\hline filtrage & 1 & 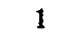 \\
\hline $\begin{array}{l}\text { dispositif de } \\
\text { reconnaissance }\end{array}$ & 1 & \\
\hline équation & 2 & 2 \\
\hline
\end{tabular}

NOM

distance

raisonneur

matrice

foncteur

tri

treillis

parcours

variable

mode

probabilité
X D TOTAL

$\begin{array}{ll}2 & 2 \\ 1 & 1 \\ 1 & 1 \\ 1 & 1 \\ 1 & 1 \\ 1 & 1 \\ 1 & 1 \\ 2 & 2 \\ 1 & 1 \\ 1 & 1\end{array}$




\section{TABLEAU 10}

\section{BASES POUR LESQUELLES LE MODIFICATEUR APPARAÎT}

SOUS LES FORMES X ET D

$\begin{array}{lllc}\text { NOM } & \text { X } & \text { D } & \text { TOTAL } \\ \text { algèbre } & 1 & 1 & 2 \\ \text { calcul } & 1 & 1 & 2 \\ \text { cercle } & 1 & 1 & 2 \\ \text { chaîne } & 1 & 2 & 3 \\ \text { distribution } & 1 & 1 & 2 \\ \text { espace } & 1 & 1 & 2 \\ \text { formalisme } & 1 & 1 & 2 \\ \text { grammaire } & 2 & 1 & 3 \\ \text { logique } & 5 & 2 & 7 \\ \text { modèle } & 2 & 1 & 3 \\ \text { méthode } & 1 & 1 & 2 \\ \text { procédure } & 2 & 1 & 3 \\ \text { réseau } & 4 & 1 & 5 \\ \text { système } & 5 & 2 & 7 \\ \text { sémantique } & 4 & 1 & 5\end{array}$


RÉSUMÉ: Les noms propres et leurs dérivés dans le vocabulaire de l'intelligence artificielle - Cet article présente les résultats d'une étude qui porte sur un sujet relativement peu exploré par les terminologues, à savoir la part des noms propres et de leurs dérivés dans la formation des vocabulaires techniques et scientifiques. Nous nous intéressons en particulier dans cet article aux onomastismes qui font partie du vocabulaire de l'intelligence artificielle. L'article se divise en trois parties. Nous présentons tout d'abord les matrices morphosyntaxiques qui composent les onomastismes de l'intelligence artificielle. Nous étudions ensuite les unités terminologiques complexes onomastiques du point de vue du nom propre et de son référent. Enfin, nous examinons ces mêmes unités du point de vue de leur déterminé.

\section{ABSTRACT: Proper Names and Their Derivatives in Artificial} Intelligence Terminology - This article presents the results of a study on a topic which has received relatively little attention from terminologists: the place of proper names and their derived forms in the formation of scientific and technical terms. The study focusses on the field of artificial intelligence: the morphosyntactic patterns of onomastic forms used in the field; the complex onomastic terminological units seen from the point of view of the proper noun and its referent; and finally, these forms as examined from the point of view of their base unit. 